\title{
Rotational properties of asteroids, comets and TNOs
}

\author{
Alan W. Harris ${ }^{1}$ and Petr Pravec ${ }^{2}$ \\ ${ }^{1}$ Space Science Institute, Boulder, CO 80301, USA \\ email: awharris@spacescience.org \\ ${ }^{2}$ Astronomical Institute, Academy of Sciences of the Czech Republic, \\ Fričova 1, CZ-25165 Ondřejov, Czech Republic \\ email: ppravec@asu.cas.cz
}

\begin{abstract}
Over the past 25 years the number of reliably determined rotation rates of asteroids has increased by an order of magnitude, from 157 in 1979 to 1686 in 2005 . As the numbers have increased, various special classes and features have emerged. Asteroids larger than $\sim 50 \mathrm{~km}$ diameter have a dispersion of spin rates that is well represented by a single Maxwellian distribution. Smaller asteroids have a more dispersed distribution, with both slow and fast spinning populations. We see a "spin rate barrier" in the size range of 1-10 km diameter that suggests that even rather small asteroids are "rubble piles". Among the very slow rotators are some (but not all) that are "tumbling" in non-principal axis rotation states. Among the smallest asteroids (less than a few hundred $m$ diameter) are some that spin dramatically faster than the "spin barrier", indicating that they must have some tensile strength rather than consisting of loose regolith. In the last few years it has been recognized that the spins of asteroids smaller than a few tens of $\mathrm{km}$ diameter are affected by radiation pressure torques that tend to either speed up or slow down asteroid spin rates, thus providing an explanation for the dispersion of small asteroid spins, and also their non-random axis orientations. Lightcurves have also revealed the presence of binary asteroids among both Near-Earth and Main-Belt populations. Automated robotic observatories and next-generation survey instruments promise to increase the rate of production of asteroid lightcurves so that we may soon have tens of thousands of lightcurve results, extending down to even smaller sizes. In contrast, there are only about 20 rotation rates known for comets, and 15 for TNOs. Very little can be said from such meager statistics; the mean spin rate of TNOs appears to be comparable to that of asteroids, without extremes of fast or slow rotation; the mean spin rate of comets appears to be a bit slower than asteroids, perhaps due to lower mean density, and there may be an excess of slow rotators, probably due to gas jetting effects. The future is promising for studies of these objects as larger telescopes become available to do photometry to fainter magnitudes, so that comet nuclei can be studied at greater heliocentric distance with less coma interference, and more TNOs can be observed.
\end{abstract}

Keywords. minor planets, asteroids, Kuiper Belt

\section{Introduction}

At the time of the first Asteroids conference and book in 1979, the total number of reliably determined rotation periods was about 157 . In the 26 years since that time, the number has grown steadily to the present number of 1686 (Figure 1). In 1979, only a few general trends and properties could be discerned (Figure 2): that the mean rotation rate was nearly constant over size with possibly just a slight dip in the mid-size range, and that there might be differences in mean rotation rate among taxonomic classes, most notably the M-class, which seemed to have shorter rotation periods on average.

The current data set consists of 1686 reliably determined rotation rates of minor planets, including NEAs, Trojans, Centaurs, TNOs, and a few "transition objects" such as 


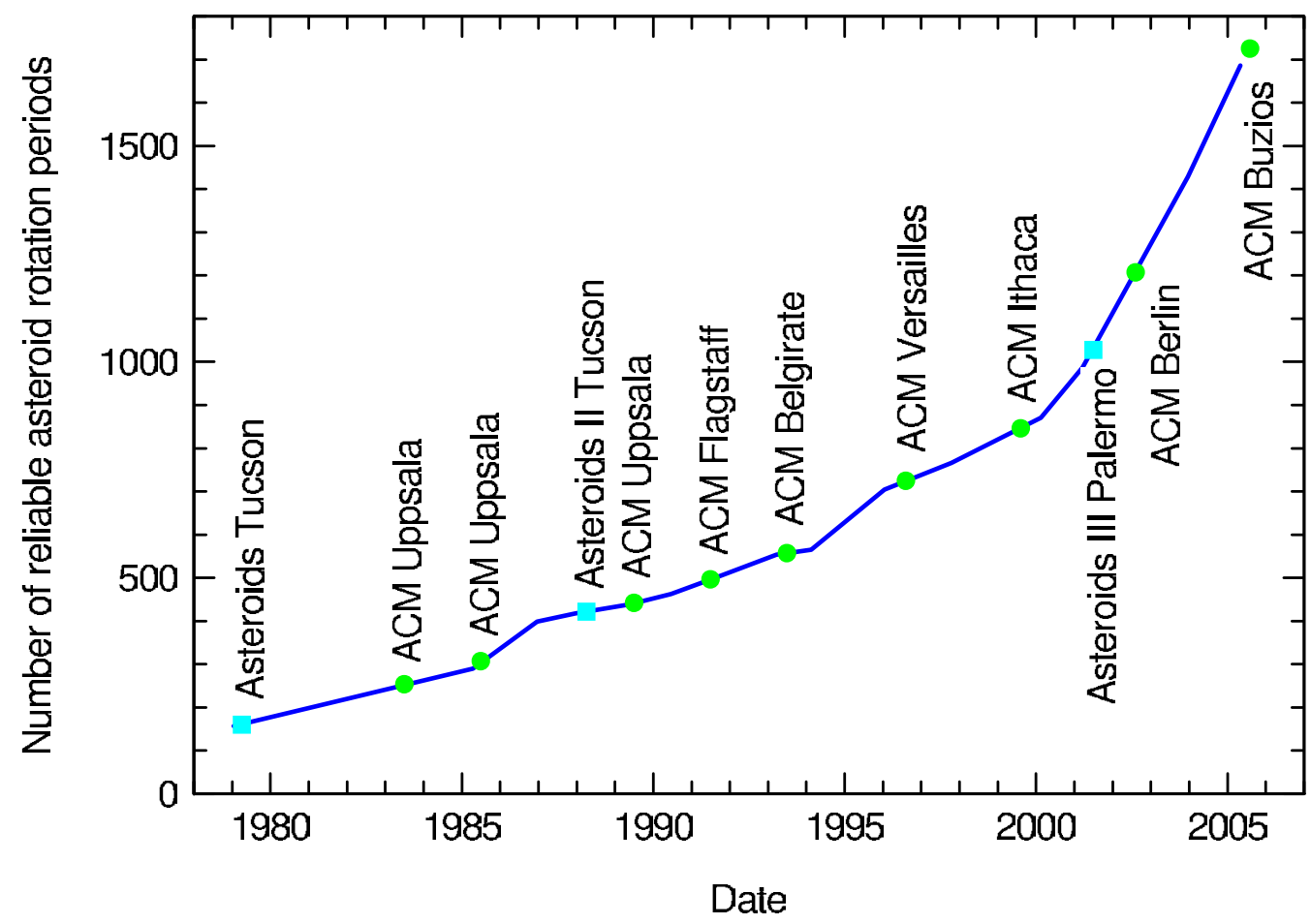

Figure 1. Number of reliably determined rotation rates of asteroids from 1979 to 2005.

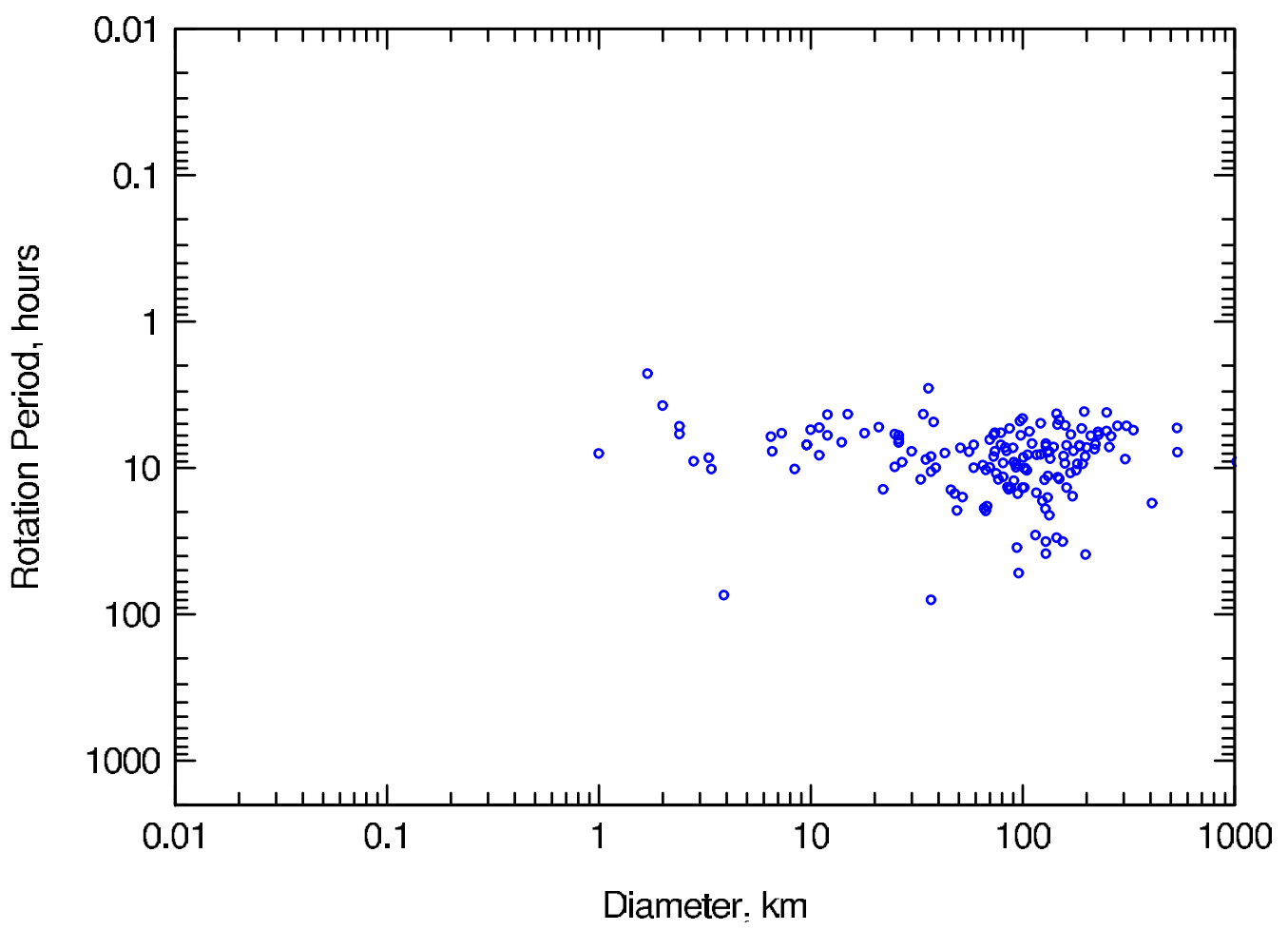

Figure 2. Rotation rate versus diameter for 157 asteroid rotations known in 1979. 


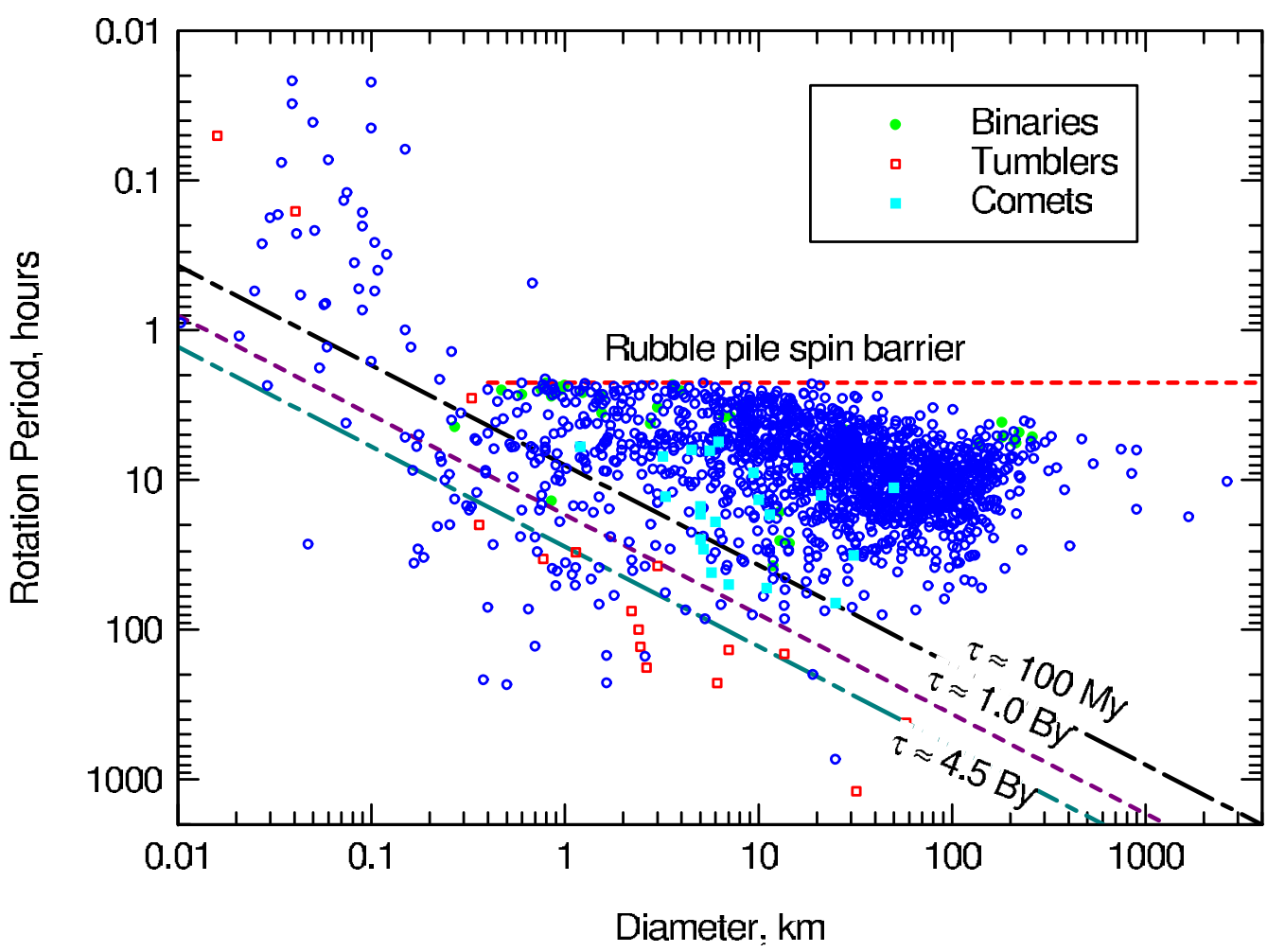

Figure 3. Rotation rate versus size for asteroids, comets and TNOs as of 2005. See text for details.

(2060) Chiron, which are also designated as "comets". Figure 3 is a plot of those rotations versus diameter of objects. The scale has been expanded a bit to include recent additions of (50000) Quaoar and (90377) Sedna, both likely larger than (1) Ceres, and we include the 20 or so comet nuclei with reasonably reliably determined rotation periods. A considerable amount of structure is apparent, which we will describe in the sections that follow. For references to the results, see Pravec et al. (2002) and references cited therein.

\section{Rotations of large asteroids}

Asteroids larger than a few tens of kilometers in diameter spin with a mean rotation period around 10 hours, with some minor variation with size. We have done a running-box calculation of the mean rate versus diameter, and then normalized individual rotation rates to the mean for that size. Thus, if an asteroid of a size where the mean spin period is 10 hours has a rotation period of 8 hours, it's normalized spin frequency is 1.25 (inverse period, compared to the mean). But in a size range where the mean spin period is 8 hours, the same 8-hour period would correspond to a normalized spin frequency of 1.0. Figure 4 is a histogram of normalized spin frequencies of large asteroids, with a 3-dimensional Maxwellian distribution appropriately normalized for comparison. The quality of this fit suggests that for asteroids larger than $\sim 50 \mathrm{~km}$ diameter, the dispersion of spin rates is as one would expect for a collisionally relaxed distribution, and in particular that the dispersion is close to isotropic in three dimensions, that is, there is not a significant 


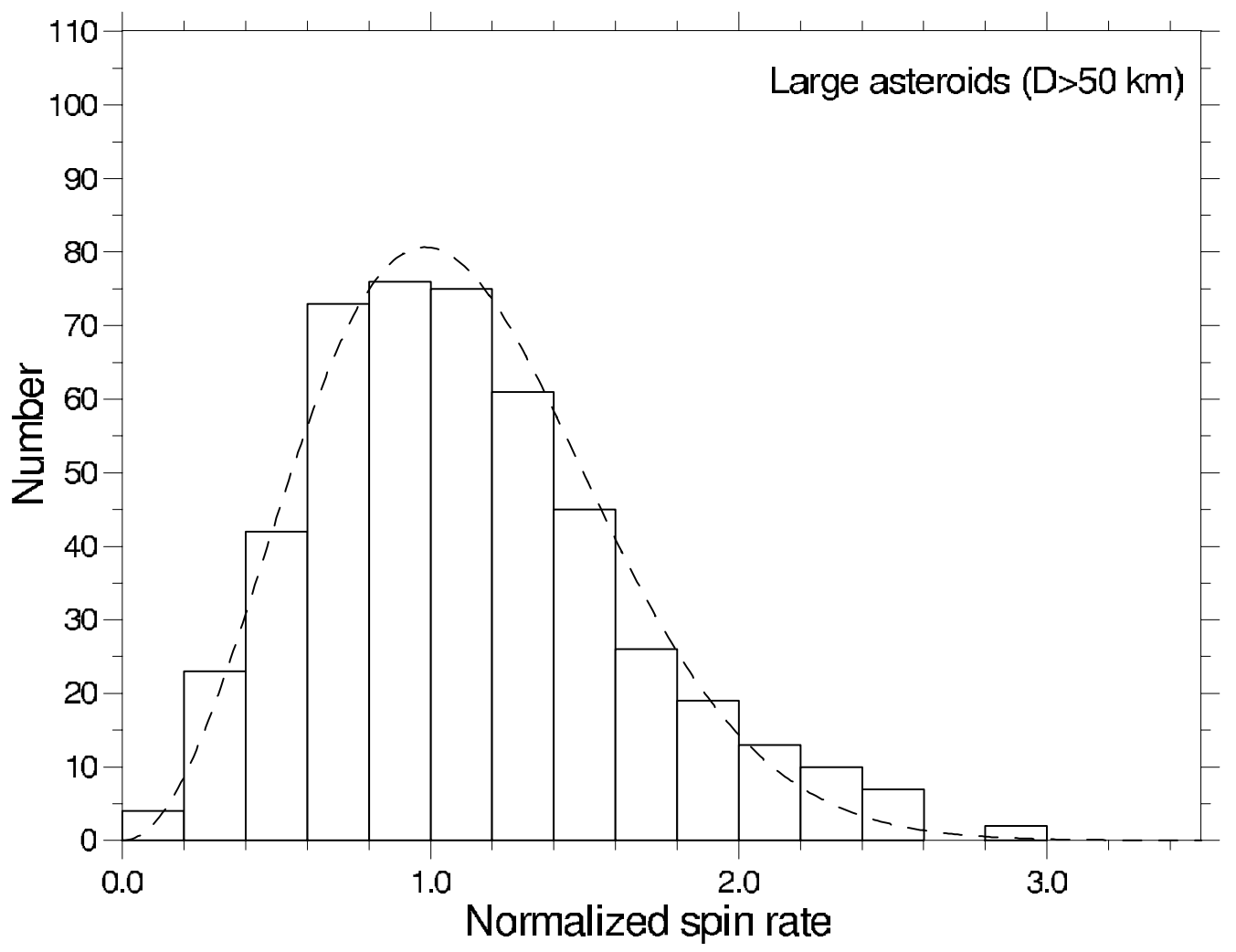

Figure 4. Spin rate distribution of large asteroids.

preferred axis alignment among larger asteroids. The small excess at $f /\langle f\rangle$ greater than 2.2 is due to faster rotating M-type asteroids.

\section{Rotations of smaller asteroids - YORP alteration}

Beginning in the range of about $50 \mathrm{~km}$ diameter, the distribution of spins deviate increasingly with decreasing size from a simple Maxwellian, becoming almost bimodal with fast and slow spins among asteroids under $10 \mathrm{~km}$ diameter. Among fast rotators, in the size range from $\sim 1-10 \mathrm{~km}$ diameter there appears to be a "barrier" to spins faster than $\sim 11$ cycles/day $(\sim 2.2$ hours period), as is readily apparent in Figure 3 . This is about the spin period at which centrifugal force at the equator equals the acceleration of gravity for a near-spherical body of expected asteroidal density in the range of $2-$ $3 \mathrm{~g} / \mathrm{cm}^{3}$. Thus we infer that the appearance of this "barrier" suggests that even such small asteroids are in some sense "rubble piles" with no substantial tensile strength. The stability limits and equilibrium shapes of rubble piles have been further studied by Holsapple (2001, 2004), and Richardson et al. (2005). As Holsapple (2004) correctly points out, the evidence is circumstantial: asteroids spinning slower than the limit may have tensile strength, we can only show that they don't need it to maintain their shapes with their given spin rates.

Below a few hundred meters in diameter, asteroids no longer "obey the speed limit", with indeed a majority spinning faster. These asteroids must be "monolithic" in the sense of possessing some material strength. It has been amply pointed out that, due to their very small size, only very modest material strength is needed to resist the centrifugal 
force of their spins, even for periods as short as a minute or so. Even the softest rock or soft clay, if not fractured, would have enough strength to retain coherence as a single body.

In the size range from a few tens of $\mathrm{km}$ diameter and extending down to the smallest observed asteroids, we see a small population, of order $10 \%$ of the total, that are very slow rotators, lying statistically well outside of a Maxwellian distribution of spins. The distribution of these spin rates is approximately uniform with spin rate, that is, the cumulative number, $N(<f)$, spinning slower than a frequency $f$ is proportional to $f$ (Harris 2002). This is essentially the distribution one might expect from a retarding force that acts like sliding friction, or for that matter tidal friction, where the magnitude of the retarding force does not depend on velocity, in this case spin frequency $f$. Rubincam (2000) proposed that thermal re-radiation from irregularly shaped bodies such as asteroids could provide the needed mechanism for spin-up as well as spin-down. Building on Rubincam's formalism, Vokrouhlický et al. (2003) showed that spin rate and axial alignments of Koronis family members (Slivan et al. 2003) provide an unmistakable "fingerprint" of so-called YORP (Yarkovsky-O'Keefe-Radzievskii-Paddack) spin evolution. While the Koronis family members provided the diagnostic evidence to prove the effect of YORP evolution, it is by no means confined to asteroids in that family, but is ubiquitous among asteroids smaller than a few tens of $\mathrm{km}$ in diameter and provides a new paradigm to explain both fast and slow spins as well as non-random spin axis alignments and possibly even shapes and binary configurations of small asteroids.

While YORP provides a mechanism for slowing down spins of some asteroids it leaves a couple questions unanswered. Many of the slowest-spinning asteroids are "tumbling", that is, in a state of non-principal axis rotation. This is in some sense not unexpected, as the damping time scale to principal axis rotation becomes long compared to asteroid ages for small and slow spinning asteroids (Burns \& Safronov 1973; Harris 1994). We plot lines of constant damping time scale in Figure 3. Confirmed tumblers lie mostly below lines of damping time scale commensurate with their expected ages (smaller asteroids are expected to be younger due to collisional disruptions). However, if YORP slows an asteroid gently over a long time from a previously faster spin, one would expect the wobble from the last disruptive collision to have damped while the asteroid was spinning fast, leaving the slowed asteroid in a state of principal-axis rotation. We see some evidence that this is so in that not all very slowly spinning asteroids are tumbling. Most of those below the long-age lines have insufficient data to say if they are tumbling or not, but a few are rather definitely determined to have little if any non-principal axis spin. Perhaps these are asteroids that were damped to principal axis spins before YORP slowed the spins, and have suffered no further excitation. Among the largest slow-spinning asteroids (e.g. (288) Glauke, (253) Mathilde), we can calculate the expected excitation of angular momentum from sub-catastrophic collisions in the main belt and show that this is sufficient to excite "tumbling." However, among smaller asteroids, YORP is so powerful that collisional excitation does not appear sufficient to excite tumbling faster than spin rate is damped. Perhaps in these cases YORP de-spins the asteroid before the tumbling component of spin has had time to damp.

Another puzzle is why YORP de-spinning does not progress all the way to spin-orbit synchronicity, as does tidal friction in some cases. Collisional excitation may provide the answer to that as well for the larger de-spun asteroids, but as in the case of tumbling, YORP appears to be too powerful for collisions to make much difference among asteroids smaller than a few $\mathrm{km}$ in diameter. Further study of these competing processes is needed.

Returning briefly to the other end of the spin distribution, YORP is fully capable of spinning up small asteroids of diameters less than $10 \mathrm{~km}$ to rates up against the "spin 
barrier", and among sub-km sized asteroids, even to the extremely fast spins observed in this size range. This raises the question of what happens when YORP spins a rubble pile asteroid up to speeds where it can no longer maintain coherence as a single body.

\section{Binary Asteroids}

After decades, even centuries, of speculation and searching, satellites of asteroids have been found, and are now being discovered at a rate of about one a month by a variety of techniques, including radar, space based imaging (HST), ground-based (mostly adaptive optics) imaging, and lightcurve monitoring (Merline et al. 2002; Pravec et al. 2006; Richardson \& Walsh 2006).

Among the earliest discovered binaries were near-Earth asteroids, generally less than one or two $\mathrm{km}$ in diameter, with satellites typically half the diameter of the primary or smaller, and in orbits not synchronously locked to the primary spin (with one exception, (69230) Hermes, which has two nearly equal components and a tidally locked spin-orbit period of 13.89 hours). The prevalence of binaries among NEAs (estimated at $\sim 15 \%$ of the total population by Merline et al. (2002), and Pravec et al. (2006)) and the apparent lack of similar numbers in the main belt suggests that the mechanism of formation might be tied to planet-crossing. The tidal disruption of comet Shoemaker-Levy 9 by Jupiter provided an obvious visual analogy at about the same time as the discovery of the first binaries (see Merline et al. (2002) for a discussion of origin scenarios and references). In the last year or two, as we obtain lightcurves of smaller and smaller main-belt asteroids, we are discovering binaries among the smaller members of the main belt (e.g., Ryan et al. 2004; Warner et al. 2005). Indeed, preliminary estimates of the frequency of binaries among MBAs smaller than $\sim 10 \mathrm{~km}$ diameter may be nearly as great as the frequency of binaries among somewhat smaller NEAs. This calls into question the idea that the formation of binaries of this sort is dominantly by tidal interaction with planets.

In Table 1 we list the properties of selected binaries (we have not included TNO binaries, which seem to have formed by other means and have little to do with rotational properties). The four columns following the object identifications list the diameter of the primary, the ratio of diameter of secondary to diameter of primary, the rotation period of the primary, and the orbit period of the secondary. The last two columns are a measure of the total angular momentum of the system, including spin and orbital angular momenta, which we call $\alpha$, in dimensionless units. The unit used is equal to the angular momentum of a homogeneous sphere of mass equal to that of the binary (primary plus secondary) if it were spinning at a rate equal to the surface orbit frequency about the sphere - that is, a rate such that a test particle on the equator would just levitate off the surface with gravity balanced by centrifugal force. This necessarily involves the density of the bodies, which is generally not known, so we tabulate values of angular momentum for densities of 2.0 and $3.0 \mathrm{~g} / \mathrm{cm}^{3}$. A spinning, homogeneous fluid takes the form of an oblate (so-called Maclaurin) spheroid for values of $\alpha<0.76$. In the range $0.76<\alpha<0.975$, the fluid takes on the form of a triaxial prolate ellipsoid, known as a Jacobi ellipsoid. At a value of $\alpha$ greater than this, the fluid fissions into two orbiting bodies (see Weidenschilling 1981 for a discussion relating to asteroid spins). Asteroids, however, are not fluids. In defining the "rotational speed limit", Harris (1996) in effect took $\alpha=1.0$ as the limit for stability of a "rubble pile". More recently, Holsapple (2001, 2004) has refined this limit for realistic unconsolidated materials, and finds that stability can be maintained up to values of $\alpha$ of about 1.3. If one imagines starting from a spherical rubble pile and adding angular momentum, at a value of $\alpha$ of about 0.9 , the figure will start to "landslide" into a Jacobi-like form, becoming more and more elongate until it reaches an elongation 
Table 1. Properties of some Asteroid binaries

\begin{tabular}{|c|c|c|c|c|c|c|}
\hline & $D_{P}$ & $D_{S} / D_{P}$ & $P_{\text {rot }}$ & $P_{\text {orb }}$ & $\begin{array}{c}\alpha \\
\rho=2\end{array}$ & $\rho=3$ \\
\hline \multicolumn{7}{|l|}{ NEA binaries } \\
\hline 3671 Dionysus & 1.5 & 0.20 & 2.71 & 27.74 & 0.90 & 0.74 \\
\hline $351071991 \mathrm{VH}$ & 1.2 & 0.38 & 2.62 & 32.66 & 1.11 & 0.98 \\
\hline 65803 Didymos & 0.8 & 0.22 & 2.26 & 11.91 & 1.06 & 0.88 \\
\hline $660631998 \mathrm{RO} 1$ & 0.8 & 0.48 & 2.49 & 14.54 & 1.20 & 1.09 \\
\hline 663911999 KW4 & 1.2 & 0.35 & 2.77 & 17.44 & 0.98 & 0.85 \\
\hline 69230 Hermes & 0.6 & 0.90 & 13.89 & 13.89 & 1.21 & 1.27 \\
\hline 859381999 DJ4 & 0.4 & 0.50 & 2.51 & 17.73 & 1.25 & 1.15 \\
\hline 887102001 SL9 & 0.8 & 0.28 & 2.40 & 16.40 & 1.04 & 0.87 \\
\hline 1994 AW1 & 1.0 & 0.49 & 2.52 & 22.30 & 1.27 & 1.17 \\
\hline 1996 FG3 & 1.5 & 0.31 & 3.59 & 16.14 & 0.75 & 0.65 \\
\hline 1999 HF1 & 3.5 & 0.23 & 2.32 & 14.03 & 1.04 & 0.86 \\
\hline 2000 DP107 & 0.8 & 0.41 & 2.77 & 42.20 & 1.15 & 1.04 \\
\hline 2000 UG11 & 0.2 & 0.50 & 4.44 & 18.40 & 0.93 & 0.88 \\
\hline 2002 CE26 & 3.0 & 0.07 & 3.29 & 16.00 & 0.71 & 0.58 \\
\hline 2003 YT1 & 1.0 & 0.18 & 2.34 & 30.00 & 1.02 & 0.84 \\
\hline 54071992 AX & 3.9 & 0.20 & 2.55 & 13.52 & 0.94 & 0.78 \\
\hline $2005 \mathrm{AB}$ & 1.1 & 0.24 & 3.34 & 17.93 & 0.75 & 0.63 \\
\hline 5381 Sekhmet & 1.0 & 0.30 & 2.7 & 12.00 & 0.94 & 0.79 \\
\hline 1990 OS & 0.3 & 0.15 & 3. & 21.00 & 0.79 & 0.65 \\
\hline $1998 \mathrm{ST} 27$ & 0.6 & 0.13 & 3.0 & 154.36 & 0.80 & 0.66 \\
\hline \multicolumn{7}{|l|}{ MB binaries } \\
\hline 22 Kalliope & 181.0 & 0.23 & 4.14 & 85.92 & 0.65 & 0.56 \\
\hline 90 Antiope & 100.0 & 1.00 & 16.53 & 16.53 & 1.29 & 1.36 \\
\hline 617 Patroclus & 105.0 & 0.90 & 81.84 & 81.84 & 2.01 & 2.15 \\
\hline 854 Frostia & 9.0 & 0.86 & 37.56 & 37.56 & 1.54 & 1.64 \\
\hline 1089 Tama & 13.0 & 0.70 & 16.44 & 16.44 & 1.01 & 1.06 \\
\hline 1313 Berna & 10.0 & 0.90 & 25.46 & 25.46 & 1.41 & 1.49 \\
\hline 1509 Esclangona & 8.0 & 0.33 & 2.64 & 474.93 & 1.33 & 1.21 \\
\hline 3782 Celle & 6.0 & 0.43 & 3.84 & 36.57 & 0.96 & 0.89 \\
\hline 3703 Volkonskaya & 3.0 & 0.40 & 3.24 & 24.00 & 0.96 & 0.86 \\
\hline 4492 Debussy & 11.0 & 0.80 & 26.59 & 26.59 & 1.32 & 1.40 \\
\hline 5905 Johnson & 3.6 & 0.40 & 3.78 & 21.79 & 0.86 & 0.77 \\
\hline 9069 Hovland & 2.8 & 0.5 & 4.22 & 30.35 & 1.04 & 0.99 \\
\hline \multicolumn{7}{|l|}{ Planets } \\
\hline Pluto-Charon & 2302 & 0.52 & 153.29 & 153.29 & 1.10 & 1.18 \\
\hline & 12742 & 0.27 & 23.93 & 655.72 & 0.40 & 0.41 \\
\hline \multicolumn{7}{|l|}{ Non-binaries } \\
\hline 433 Eros & 17. & - & 5.27 & - & & 1.01 \\
\hline 1566 Icarus & 1.3 & - & 2.27 & - & 1.10 & 0.90 \\
\hline 1620 Geographos & 3. & - & 5.22 & - & 0.98 & 0.80 \\
\hline
\end{tabular}

axis ratio of two or three to one and a value of $\alpha$ of about 1.3. At still higher angular momentum, it should bifurcate, or perhaps shed mass from the equator in a nearly "fluid" way. Richardson et al. (2005) suggest the latter, although probably that depends on the detailed structure of the "rubble pile".

The slow spin-up of small asteroids by the YORP effect suggests a mechanism for making binaries among small asteroids, and indeed a mechanism that should be nearly as effective among small main-belt asteroids as among planet-crossing asteroids. In this scenario, as a rubble pile asteroid is spun up by the YORP effect, it might "landslide" into increasingly elongate shapes until it either sheds matter in bits and pieces to reform into a satellite, or it might spontaneously bifurcate into two co-orbiting bodies. To investigate this further, we have attempted to calculate the angular momentum of 
observed binary systems, as listed in the final two columns of Table 1 . The fact that most of the primaries are spinning very fast suggests that rotation near the critical rate is at least a requirement, if not the cause, of binary formation. It is particularly noteworthy that many of the binaries contain just about exactly the critical angular momentum for splitting into binaries. However, it is also noteworthy that quite a few binaries contain less than the critical angular momentum, so that they must have either lost some angular momentum after fissioning, or were created in some other way. It also seems remarkable that almost no binaries have significantly more than the critical angular momentum. Thus, if formed by spin-up to fission, the spin-up torque must be shut off as the binary is formed.

We call attention to the few entries at the end of the table. The Pluto/Charon binary system fits nicely into the size ratio and angular momentum profile to have been formed by fission followed by angular momentum conserving tidal evolution to its present state. But certainly it was not spun up by YORP! The Earth-Moon system is a factor of two deficient in angular momentum for the moon to have formed by fission, a fact known for more than a century. And finally, we list a few non-binaries, very fast-spinning single asteroids. These single bodies contain about the same critical angular momentum as many of the binary systems, and more than some of them. Why do these asteroids remain single bodies while others with the same angular momentum are binaries?

\section{Concluding remarks}

So far we have said very little about the spins of comets, Centaurs, and TNOs. The number of reliably measured spins of these classes of objects is too few to make detailed comparisons or to draw conclusions. Comet nuclei spins (plotted in Figure 3) appear more dispersed than "Maxwellian", and maybe a bit slower on average than asteroids. This is consistent with presumed lower density and with outgassing torques which could tend to spin them up or down, in the same way YORP affects small asteroid spins. Centaurs and TNOs are plotted in Figure 3 but not with special symbols, so they cannot be discerned from the asteroids. However, they appear to have a somewhat longer mean rotation period than asteroids, but otherwise similar statistics, consistent with "Maxwellian", but with too few measured values to critically test that hypothesis.

We conclude by mentioning some avenues of investigation that seem ripe for pursuit in the study of rotations of small bodies:

- Robotic and/or remote controlled telescopes with CCD cameras provide an ideal means to obtain vastly greater volumes of data than was possible using "hands on" observations with photoelectric or even CCD systems. This opens up the possibility of conducting surveys of many more objects with more densely sampled lightcurves, going to smaller sizes and looking for complex lightcurves revealing binary systems and "tumbling" asteroids.

- The authentication of young asteroid families by tracing back orbits to a common nodal alignment (Nesvorný et al. 2002; Nesvorný \& Bottke 2004) provides us with the opportunity to study asteroid spins presumably unevolved since the time of the parent asteroid break-up. Small members of such families (Karin, for example) should not show a bimodal spin distribution nor anisotropic spin axis alignments that have been demonstrated among the rest of the Koronis family. One might even be able to age date some of the medium age families (those too old to date by nodal alignment) by determining the degree of YORP alteration among members of the family.

- We are on the verge of directly measuring the slow-down of at least one asteroid (25143 Itokawa; Vokrouhlický et al. 2004). Because a change in rotation rate results in an 
accumulating shift in rotation phase, an exceedingly small deceleration can be detected over years of observation, so directly measuring the YORP change in rotation rates of small asteroids is entirely feasible and we can expect to obtain such results from detailed observations over the coming few years or decade.

- Kaasalainen (2004) has recently presented a method for extracting shape and pole information from sparse photometry such as will be obtained for thousands of asteroids by the next generation of sky surveys (Pan-STARRS, LSST, GAIA). Applying this method to data from next-generation surveys holds the promise of increasing the number of asteroids for which we have rotation and shape information by two orders of magnitude or more. One can only guess what "fine structure" might emerge from a plot like Figure 3 with 100 times more "resolution".

- The present and ever-increasing flood of lightcurve data is leading to binaries galore. Within a year or two there will likely be more than 100 known, so serious theoretical and modeling efforts to understand their origin and evolution can get underway. The study is just beginning.

\section{Acknowledgements}

The work at Space Science Institute (A.W.H.) was supported by grant NAG5-13244 from the NASA Planetary Geology-Geophysics Program. The work at Ondřejov (P.P.) was supported by the Grant Agency of the Czech Republic, Grant 205/05/0604.

\section{References}

Burns, J.A. \& Safronov, V.S. 1973, Mon. Not. Roy. Astron. Soc. 165, 403

Harris, A.W. 1994, Icarus 107, 209

Harris, A.W. 1996, Lunar $\&$ Planetary Sci. XXVII, 493

Harris, A.W. 2002, Icarus 156, 184

Holsapple, K.A. 2001, Icarus 154, 432

Holsapple, K.A. 2004, Icarus 172, 272

Kaasalainen, M. 2004, Astron. \& Astrophys. 422, L39

Merline, W.J., Weidenschilling, S.J., Durda, D.D., Margot, J.-L., Pravec, P., \& Storrs, A.D. 2002, in: W.F. Bottke Jr., A. Cellino, P. Paolicchi, \& R.P. Binzel (eds.), Asteroids III (Tucson: University of Arizona Press), p. 289

Nesvorný, D., Bottke, W.F., Levison, H., \& Dones, L. 2002, Nature 417, 720

Nesvorný, D. \& Bottke, W.F. 2004, Icarus 170, 324

Pravec, P., Harris, A.W., \& Michałowski, T. 2002, in: W.F. Bottke Jr., A. Cellino, P. Paolicchi, \& R.P. Binzel (eds.), Asteroids III, (Tucson: University of Arizona Press), p. 113

Pravec, P., \& 56 co-authors 2005, Icarus in press

Richardson, D.C., Elankumaran, P., \& Sanderson, R.E. 2005, Icarus 173, 349

Richardson, D.C. \& Walsh, K.J. 2006, Ann. Rev. Earth 8 Planetary Sci., in press

Rubincam, D.P. 2000, Icarus 148, 2

Ryan, W.H., Ryan, E.V., \& Martinez, C.T. 2004, Planetary and Space Sci. 52, 1093

Slivan, S.M., Binzel, R.P., Crespo da Silva, L.D., Kaasalainen, M., Lyndaker, M.M., \& Krčo, M. 2003, Icarus 162, 285

Vokrouhlický, D., Čapek, D., Kaasalainen, M., \& Ostro, S.J. 2004, Astron. \& Astrophys. 414, L21

Vokrouhlický, D., Nesvorný, D., \& Bottke, W.F. 2003, Nature 425, 147

Warner, B.D., Pravec, P., Harris, A.W., Galad, A., Kusnirak, P., Pray, D.P., Brown, P., Krzeminski, Z., Cooney Jr., W.R. \& 10 co-authors 2005, IAU Symp. 229 Abstracts, $\partial$. 89

Weidenschilling, S.J. 1981, Icarus 46, 124 